\title{
Recognizing emotion from Turkish speech using acoustic features
}

\author{
Caglar Oflazoglu and Serdar Yildirim*
}

\begin{abstract}
Affective computing, especially from speech, is one of the key steps toward building more natural and effective human-machine interaction. In recent years, several emotional speech corpora in different languages have been collected; however, Turkish is not among the languages that have been investigated in the context of emotion recognition. For this purpose, a new Turkish emotional speech database, which includes 5,100 utterances extracted from 55 Turkish movies, was constructed. Each utterance in the database is labeled with emotion categories (happy, surprised, sad, angry, fearful, neutral, and others) and three-dimensional emotional space (valence, activation, and dominance). We performed classification of four basic emotion classes (neutral, sad, happy, and angry) and estimation of emotion primitives using acoustic features. The importance of acoustic features in estimating the emotion primitive values and in classifying emotions into categories was also investigated. An unweighted average recall of $45.5 \%$ was obtained for the classification. For emotion dimension estimation, we obtained promising results for activation and dominance dimensions. For valence, however, the correlation between the averaged ratings of the evaluators and the estimates was low. The cross-corpus training and testing also showed good results for activation and dominance dimensions.
\end{abstract}

Keywords: Turkish emotional speech database; Emotion recognition; Emotion primitives estimation; Cross-corpus evaluation

\section{Introduction}

Recognizing the emotional state of the interlocutor and changing the way of communicating accordingly play a crucial role for the success of human-computer interaction. However, many technical challenges need to be resolved before integrating a real-time emotion recognizer into human-computer interfaces. These challenges include, as in any pattern recognition problem, data acquisition and annotation, feature extraction and finding the most salient features, and building a robust classifier. In this paper, we address each of these problems in the context of emotion recognition from Turkish speech and perform a cross-corpus evaluation.

The lack of data is a major challenge in emotion recognition. Even though great efforts have been made to collect emotional speech data in recent years, there is still a need for emotional speech recordings to cope with the

*Correspondence: serdar@mku.edu.tr

Computer Engineering Department, Mustafa Kemal University, Iskenderun, 31200, Hatay, Turkey problem of data sparseness. One way to obtain emotional speech data is to use human subjects reading utterances, generally with a certain number of pre-determined and emotionally neutral sentences, in specified emotional states. Berlin database of emotional speech [1], Danish Emotional Speech [2], LDC Emotional Prosody Speech and Transcripts [3], and Geneva Multimodal Emotion Portrayals (GEMEP) [4] are examples of studio-recorded emotional speech databases.

Even though studio-recorded (acted) databases provide us more balanced data in terms of the number of utterances per emotion, emotions are less natural and realistic compared to those we encounter in real life. One way to overcome this problem is to create environments so that the subjects produce the desired emotions. Sensitive Artificial Listener (SAL) [5], Airplane Behaviour Corpus (ABC) [6], Speech Under Simulated and Actual Stress (SUSAS) [7], TUM Audiovisual Interest Corpus (AVIC) [6], Interactive Emotional Dyadic Motion Capture (IEMOCAP) [8], the SEMAINE database [9], and the FAU Aibo emotion corpus [10] are examples of such

\section{望 Springer}

(C) 2013 Oflazoglu and Yildirim; licensee Springer. This is an Open Access article distributed under the terms of the Creative Commons Attribution License (http://creativecommons.org/licenses/by/2.0), which permits unrestricted use, distribution, and reproduction in any medium, provided the original work is properly cited. 
databases. For example, the FAU Aibo emotion corpus consists of $9 \mathrm{~h}$ of German spontaneous speech of 51 children interacting with Sony's pet robot Aibo. A Wizardof-Oz technique was used for data collection and then the speech data was annotated with 11 emotion categories by five annotators at word level [10]. Audio-visual recordings obtained from TV shows and movies are also used for data acquisition, e.g., Vera-Am-Mittag (VAM) database [11], Situation Analysis in Fictional and Emotional Corpus (SAFE) [12], and the Belfast Naturalistic Database [13]. For example, the VAM corpus consists of audio-visual recordings taken from German TV talk show called Vera-Am-Mittag. The corpus contains 946 spontaneous speech from 47 participants of the show. The SAFE corpus [12] contains $7 \mathrm{~h}$ of audio-visual data extracted from English fiction movies and is mainly constructed for the purpose of fear-type emotion recognition system. In this paper, we utilized Turkish movies and TV shows to obtain speech data since the emotional speech extracted from movies is more realistic than studio-recorded emotions expressed by actors reading some pre-defined sentences.

An important requirement of most data-driven systems is the availability of annotated data. The goal of annotation is to assign a label to data. For the emotion recognition task, the annotation is needed to determine the true emotion expressed in the collected speech data. Largely motivated from psychological studies, two approaches were employed within the emotion recognition research for emotion annotation. The classical approach is to use set of emotion words (categories) to describe emotionrelated states. Even though there are ongoing debates concerning how many emotion categories exist, the emotion categories (fear, anger, happiness, disgust, sadness, and surprised) defined by Ekman [14] are commonly used in most of the studies on automatic emotion recognition. However, the main disadvantage of the categorical approach is that it fails to represent a wide range of reallife emotions. The second approach is to use continuous multidimensional space model to describe emotions. In this approach, the emotion is defined as points in multidimensional space rather than a small number of emotion categories. Dimensions in this approach are called emotion primitives. The most commonly used dimensions are valence, activation, and dominance. Valence represents negative to positive axis, activation represents calm to excited axis, and dominance represents weak to strong axis in 3D space. The most common databases such as the FAU Aibo emotion corpus, Situation Analysis in Fictional and Emotional Corpus (SAFE), Airplane Behaviour Corpus (ABC), and TUM Audiovisual Interest Corpus (AVIC) were annotated with the categorical approach. Only a few databases exist where emotions are represented by emotion primitives. Sensitive Artificial Listener (SAL) [5] and
Vera-Am-Mittag (VAM) [11] are labeled with the dimensional approach. To our knowledge, among the common databases, only a few of them includes both categorical and dimensional labeling such as IEMOCAP [8] and Belfast Naturalistic Database [15].

Many previous efforts have addressed emotion recognition by employing pattern recognition techniques using segmental and/or supra-segmental information obtained from speech [6,16-26]. Acoustic parameters of speech signal have been used extensively to separate emotional coloring present in the speech. Acoustic features are obtained from low-level descriptors (LLDs) such as pitch, energy, duration, Mel-frequency cepstral coefficients (mfcc), and voice quality parameters by applying functionals (mean, median, percentiles, etc.). Comprehensive list of LLDs and functionals is given in [27]. Linguistic information can also be used for emotion recognition especially when the speech data is spontaneous [16,18,22,25,28-31]. In this study, we only considered acoustic features and used the same feature set given in the INTERSPEECH 2010 Paralinguistic Challenge [32].

In this paper, we also performed cross-corpus evaluations where the system is trained on one corpus and tested on another. Only a few studies provide such cross-corpus results [33,34]. In [34], cross-corpus evaluation results of six well-known emotional speech databases were provided. In this work, we provided cross-corpus results using the VAM database.

This paper is organized as follows. Section 2 describes Turkish emotional speech database. Section 3 explains the feature extraction and selection procedures. Experimental setup and results are given in Section 4. Section 5 concludes the paper.

\section{Turkish emotional speech database}

In recent years, several corpora in different languages have been collected; however, Turkish is not among the languages that has been investigated in the context of emotion recognition. As an attempt to create a TURkish Emotional Speech database ${ }^{a}$ (TURES), we have recently extracted and annotated a large amount of speech data from 55 Turkish movies [35].

\subsection{Acquisition}

Collecting real-life utterances is a challenging task; hence, most of the previous studies have used speech data with studio-recorded emotions. In this study, we decided to use Turkish movies from various genres for data collection because the speech extracted from movies is more realistic than studio-recorded emotions expressed by speakers reading some pre-defined sentences. The data collection process has been done in several stages. First, the audio tracks were extracted from each movie and saved as a separate file. The movies were originally in video object (vob) 


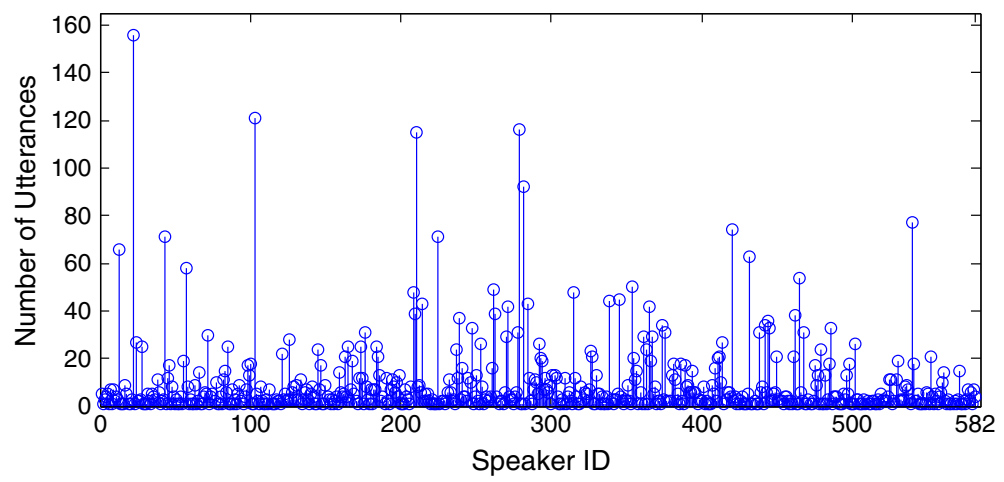

Figure 1 The number of utterances per speaker.

format that contains audio data in Dolby Digital (AC-3) (six channel) format. Then, the audio channel that contains the dialogues was separated from other audio channels for each movie and saved as a separate file at $48 \mathrm{kHz}$, 16 bit, mono, PCM-wave format. In the final stage of the data acquisition process, the audio data containing dialogue sequences were segmented into utterances manually by two native Turkish speakers. During the segmentation procedure, the utterances that are affected by background noise/music were removed from the database, resulting in 5,303 utterances. The utterances that have low intelligibility were also removed from the database. As a result, 5,100 utterances from 582 (188 females, 394 males) speakers were obtained. The average length of the utterances is $2.34 \mathrm{~s}$. The distribution of the utterances over the speakers is given in Figure 1 and Table 1. Note that having 448 of the speakers with less than ten utterances shows how diverse the database is, thus introduces a difficulty to the emotion recognition.

\subsection{Annotation of emotional content}

The annotation is needed to determine the true emotion expressed in the speech data. In this study, we employed both categorical and dimensional approaches for emotion annotation. In categorical approach, a set of emotion words are used to describe emotionrelated states. On the other hand, in the dimensional approach, the emotion is defined as points in

Table 1 Distribution of utterances over speakers

\begin{tabular}{lc}
\hline Number of utterances & Number of speakers \\
\hline$\geq 100$ & 4 \\
50 to 99 & 10 \\
25 to 49 & 39 \\
10 to 24 & 81 \\
2 to 9 & 279 \\
1 & 169 \\
\hline
\end{tabular}

multidimensional space rather than a small number of emotion categories.

The emotion in each utterance was evaluated in a listener test by a large number of annotators ( 27 university students) independently of one another. The annotators were asked to listen to the entire speech recordings (randomly permuted) and assign an emotion label (both categorical and dimensional) for each utterance. The annotators only took audio information into consideration.

\subsubsection{Categorical annotation}

Utterances were labelled in seven emotional states: happy, surprised, sad, angry, fear, neutral, and others. For each utterance, the final emotion label was computed from the majority label of the 27 annotators. The distribution of the utterances over emotion classes is given in Figure 2, and the distributions of the utterances over speakers for each emotion class are given in Figure 3. As expected, neutral is the majority of the expressed emotions.

For assessing the quality of the annotations (i.e., interannotator reliability), we used the Fleiss' kappa metric [36]. The kappa, $\kappa$, is defined as,

$$
\kappa=\frac{P_{\mathrm{a}}-P_{\mathrm{c}}}{1-P_{\mathrm{c}}},
$$

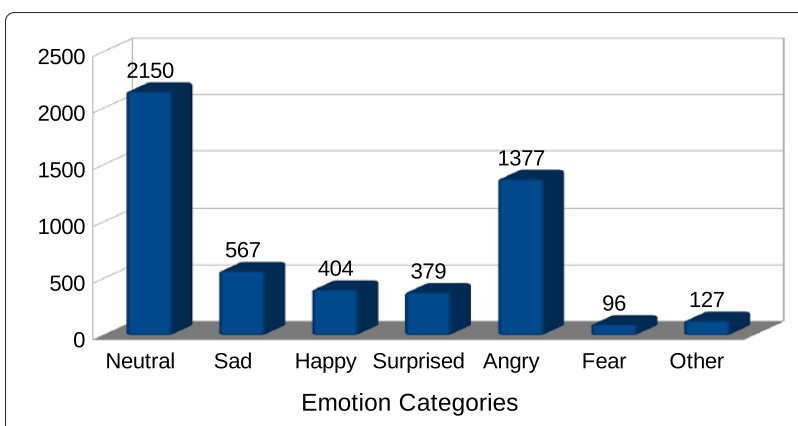

Figure 2 The number of utterances for each emotion class. 


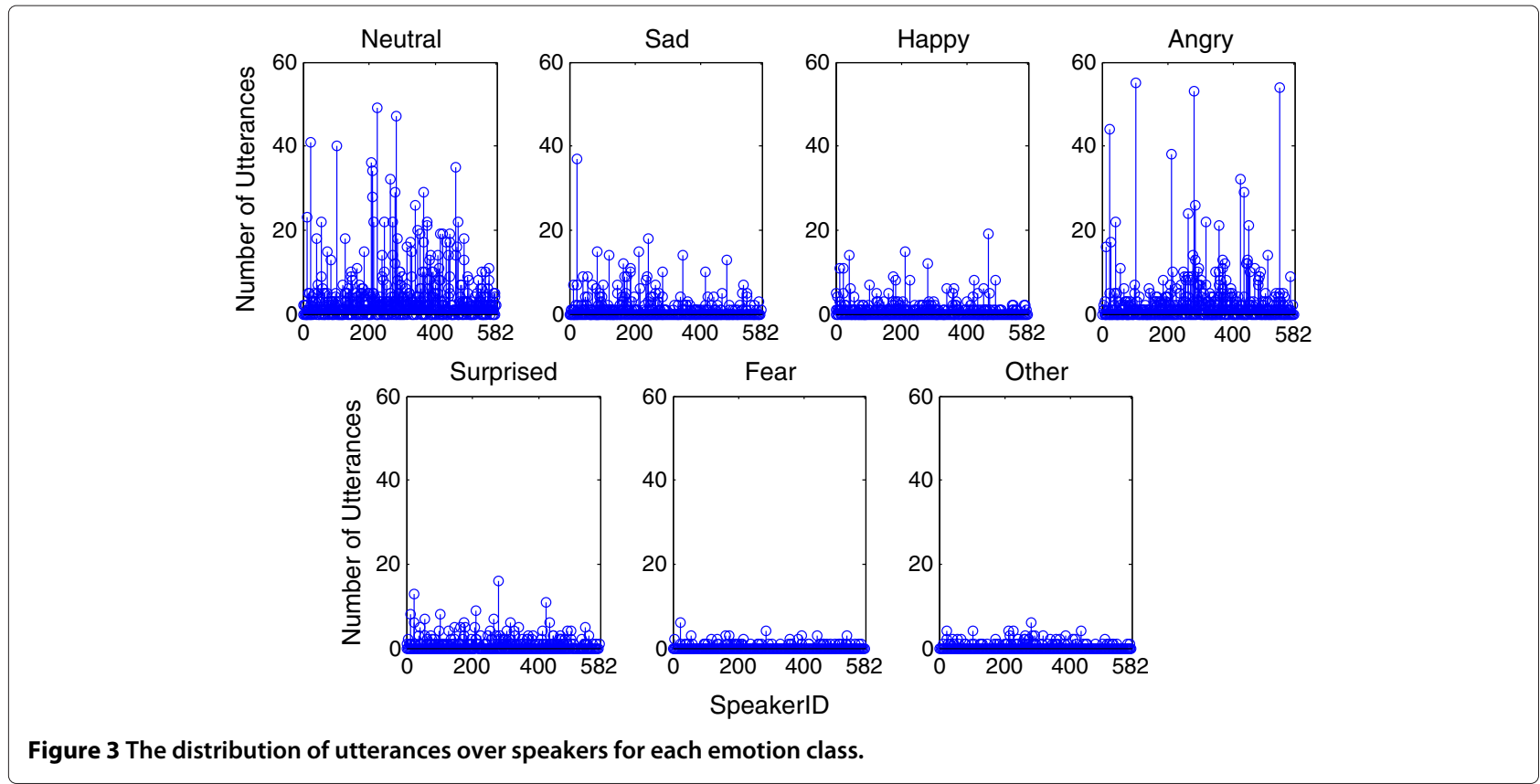

where $P_{\mathrm{a}}$ is the proportion of times that the $n$ evaluators agree, and $P_{\mathrm{c}}$ is the proportion of times we would expect the $n$ evaluators to agree by chance. The details of how $P_{\mathrm{a}}$ and $P_{\mathrm{c}}$ can be calculated are as in [36]. If there is no agreement among the evaluators, $\kappa=0$, and $\kappa=1$ when there is full agreement. The kappa score computed for the agreement level of the emotion categories between the 27 annotators is 0.32 . A score between 0.2 and 0.4 may be considered moderate inter-evaluator agreement.

\subsubsection{Annotation in 3D space}

For the emotion labelling in 3D space, we followed the same procedure proposed in [11] for emotion primitives evaluation. Self-assessment manikins (SAMs) [37] (Figure 4) were used for measuring the emotional content of each audio clip with ratings on a five-level scale between one and five for valence, activation, and dominance. Valence represents negative to positive axis, activation represents calm to excited axis, and dominance

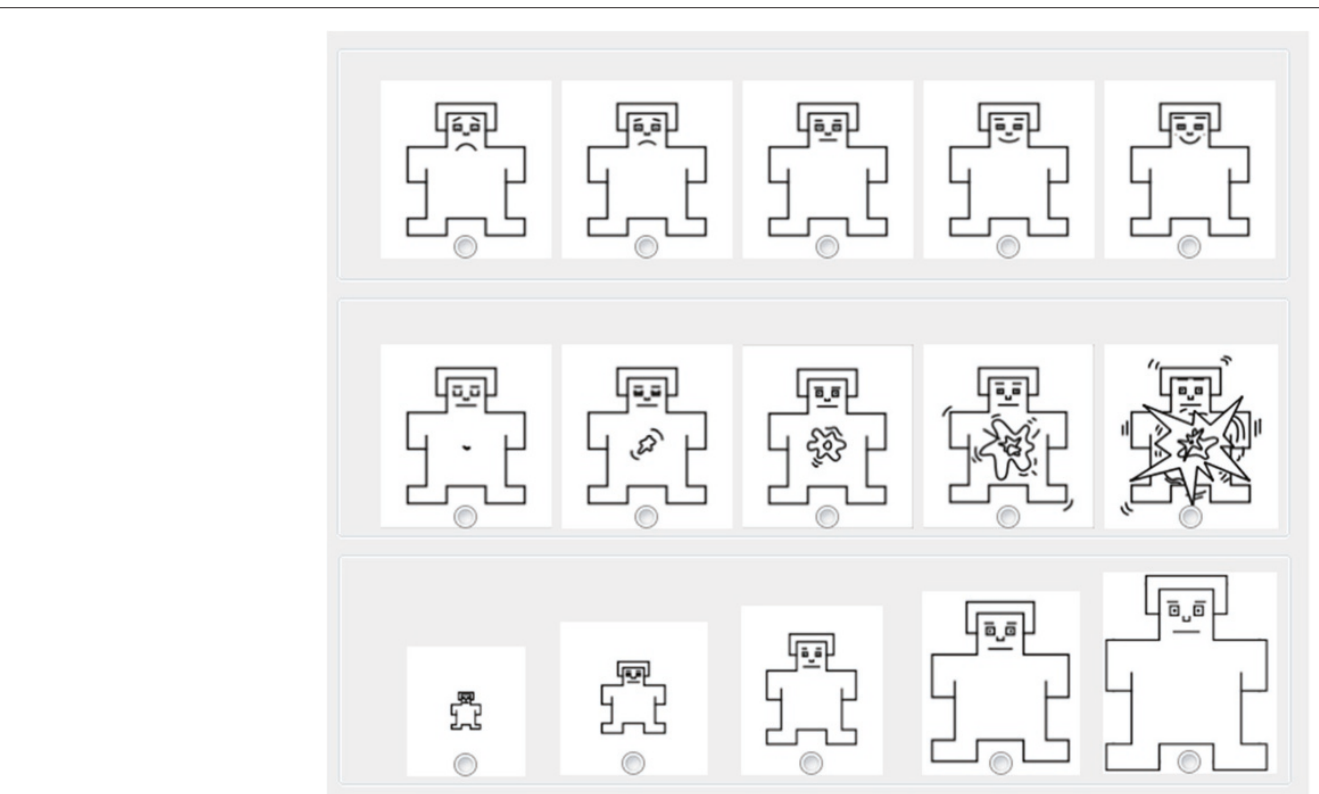

Figure 4 Self-assessment manikins [37]. First row is for valence, second row is for activation, and the third row is for dominance. 

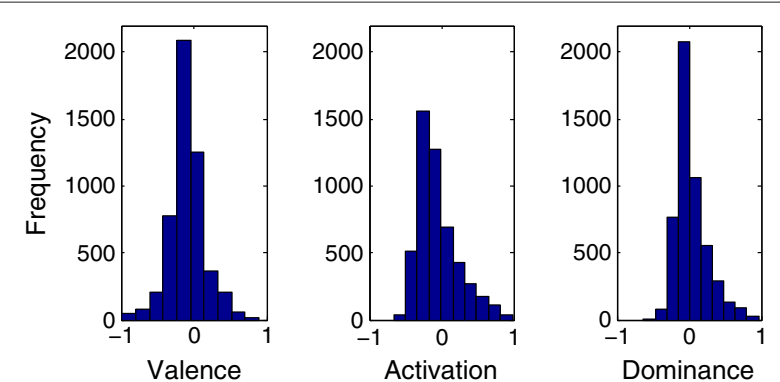

Figure 5 Histograms of the emotions in the database.

represents weak to strong axis of three-dimensional emotion space. For each utterance in the database, annotators were asked to select one of the iconic image from the corresponding row for each of three dimensions. The ratings for each emotion dimension were later transformed to unity space [-1,1] [11]. To estimate the true emotion (ground truth) of each utterance based on 27 annotators, we simply took the mean of the annotator's ratings. Figure 5 shows the histograms of the emotions based on the mean of the annotator's ratings.

To measure the agreement among the annotators, the standard deviations can be calculated for each utterance. The correlation coefficients between the annotator's ratings and the average ratings of all annotators can also be used to describe the inter-annotator reliability. In Figure 6, the distributions of the standard deviations are given in terms of box plots. The averages of standard deviations are $0.28,0.43$, and 0.39 for valence, activation, and dominance, respectively, indicating good agreement between annotators. The distribution of the correlation coefficients is given in Figure 7, and the related statistics are shown in Table 2. As can

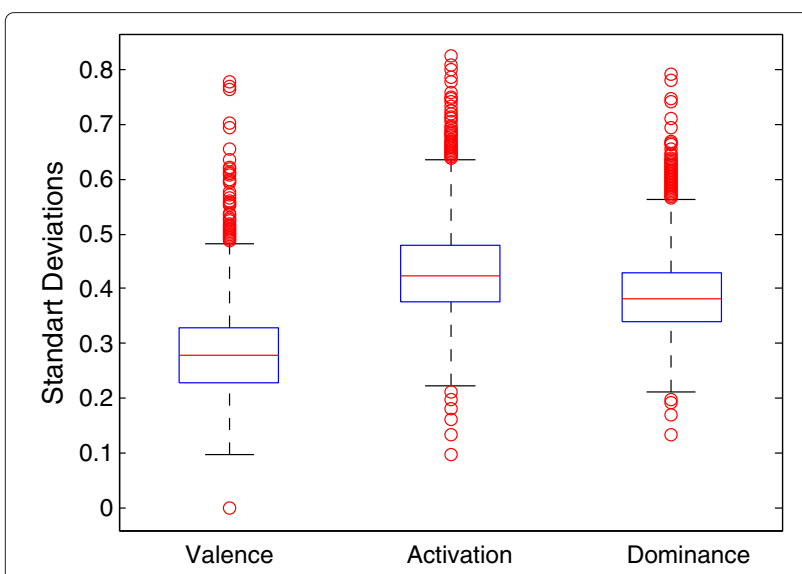

Figure 6 The distributions of the standard deviations for each emotion primitives. Utterances with low standard deviation show high inter-annotator agreement.

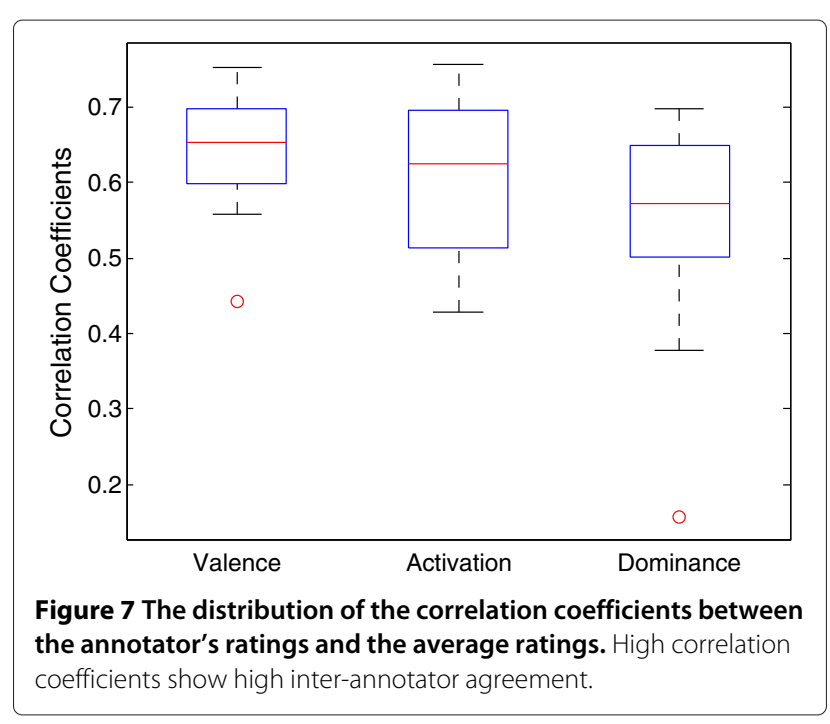

be observed from Table 2, the mean values of correlations between the annotator's ratings and the average ratings for all dimensions show also high agreement $(0.65$, 0.61 , and 0.56 for valence, activation, and dominance, respectively).

The distribution of categorical emotions in 3D space is shown in Figure 8. The class centroid, the mean, and the standard deviation of valence, activation, and dominance values of each emotion category are given in Table 3. The results show that angry speech data is negative and strong, and has high activation. Sad speech data is very negative, whereas happy speech data is very positive compared to other emotions. It is worth to note that the standard deviation values of emotion classes are high, and this might be the reason for the moderate inter-evaluator agreement in the categorical space.

\section{Acoustic features}

In this study, we used the same feature set, a set of 1,532 acoustic features based on several acoustic low-level descriptors (LLDs) and statistics (functionals), used in the INTERSPEECH 2010 Paralinguistic Challenge [32]. We extracted these features using the openSMILE toolkit [27]. The LLDs include fundamental frequency (F0), loudness, voicing probability, $0-14 \mathrm{mfcc}, 0$ to 7 logarithmic power

Table 2 Statistics from the distribution of the correlation coefficients between the annotator's ratings and the average ratings

\begin{tabular}{lllll}
\hline & Mean & Stdv & Min & Max \\
\hline Valence & 0.65 & 0.08 & 0.44 & 0.75 \\
Activation & 0.61 & 0.10 & 0.43 & 0.76 \\
Dominance & 0.56 & 0.12 & 0.16 & 0.70 \\
\hline
\end{tabular}



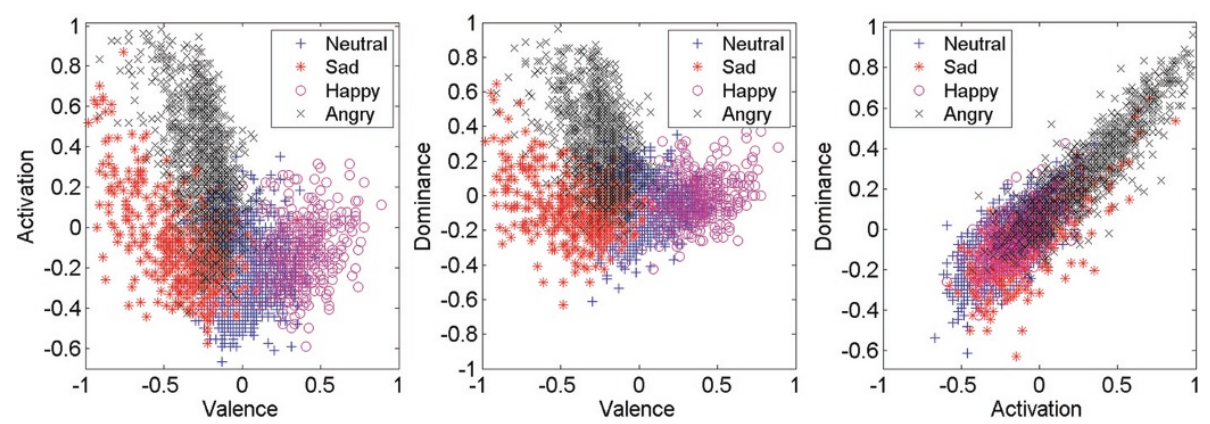

Figure 8 Distribution of emotion categories in 3D emotion space.

of Mel-frequency bands (logMelFreqBand), 0 to 7 line spectral pair frequencies computed from 8 LPC coefficients (lspFreq), and the voice quality measures (shimmer and jitter). Delta coefficients of each these LLDs are also included.

Functionals applied to descriptors are absolute position (frame) of the maximum value (maxPos), absolute position (frame) of the minimum value (minPos), arithmetic mean of the contour (amean), slope of a linear approximation of the contour (linregc1), onset of a linear approximation of the contour (linregc2), linear error computed as the difference of the linear approximation and the actual contour (linregerrA), quadratic error computed as the difference of the linear approximation and the actual contour (linregerrQ), standard deviation of the values in the contour (stddev), skewness, kurtosis, three first quartiles (quartile1: $25 \%$ percentile, quartile2: $50 \%$ percentile, and quartile3: $75 \%$ percentile), three inter-quartile ranges (iqr1-2: quartile1-quaritle2, iqr23: quartile2-quaritle3, and iqr1-3: quartile1-quaritle3), outlier-robust minimum value of the contour (percentile1.0), outlier-robust maximum value of the contour (percentile99.0), outlier robust signal range (pctlrange01 ), percentage of time the signal is above $75 \% \times$ range + minimum value (upleveltime75), and percentage of time the signal is above $90 \% \times$ range + minimum signal value (upleveltime90). A summary of the acoustic features is given in Table 4.

\subsection{Feature selection}

To identify the most salient features and thus reduce the number of feature size, we performed feature selection. For this purpose, we used correlation-based feature selection (CFS) [38]. The idea behind the correlation-based feature selection technique is to find a subset in which features are uncorrelated with each other but highly correlated to the class [38]. CFS is a filter-based subset feature evaluation algorithm that evaluates each feature subset using simple objective functions. The algorithm computes a heuristic measure of the 'merit' of the feature subset $S$ using Equation 2.

$$
\text { Merit }_{S}=\frac{k r_{\mathrm{cf}}}{\sqrt{k+k(k-1) r_{\mathrm{ff}}}}
$$

where $k$ is the number of features in subfeature space $S, r_{\mathrm{cf}}$ is the average class-feature correlation, and $r_{\mathrm{ff}}$ is the average feature-feature inter-correlation. CFS calculates $r_{\mathrm{cf}}$ and $r_{\mathrm{ff}}$ using a symmetric information gain.

Since exhaustive search through all possible feature subsets is not feasible, sub-optimal but faster search functions such as hill climbing, genetic, best first, and random are

Table 3 Comparison of emotion class centroids, mean, and standard deviations (stdv) in the 3D emotion space

\begin{tabular}{lcccccccrr}
\hline & \multicolumn{3}{c}{} & \multicolumn{2}{c}{ Valence } & \multicolumn{3}{c}{ Activation } & \multicolumn{3}{c}{ Dominance } \\
& Centroid & Mean & Stdv & Centroid & Mean & Stdv & Centroid & Mean & Stdv \\
\hline Neutral & -0.02 & -0.02 & 0.12 & -0.24 & -0.23 & 0.14 & -0.09 & -0.08 & 0.11 \\
Sad & -0.35 & -0.37 & 0.21 & -0.11 & -0.10 & 0.21 & -0.07 & -0.07 & 0.16 \\
Happy & 0.37 & 0.37 & 0.16 & -0.13 & -0.13 & 0.16 & -0.02 & -0.02 & 0.13 \\
Surprised & -0.07 & -0.06 & 0.15 & -0.13 & -0.12 & 0.17 & -0.04 & -0.04 & 0.13 \\
Angry & -0.22 & -0.23 & 0.14 & 0.26 & 0.27 & 0.28 & 0.26 & 0.28 & 0.22 \\
Fear & -0.22 & -0.24 & 0.17 & 0.04 & 0.05 & 0.21 & 0.04 & 0.04 & 0.14 \\
Others & -0.04 & -0.03 & 0.14 & -0.09 & -0.08 & 0.20 & 0.02 & 0.04 & 0.19 \\
\hline
\end{tabular}


Table 4 Overview of low-level descriptors and functionals

\begin{tabular}{lc}
\hline Low-level descriptors & Functionals \\
\hline Pitch (F0) & maxPos, minPos \\
Loudness & amean, stddev, skewness, kurtosis \\
mfcc & linregc1, linregc2, linregerrA, linregerrQ \\
logMelFreqBand & quartile1, quartile2, quartile3, iqr1-2, iqr2-3, iqr1-3 \\
IspFreq & percentile1.0, percentile99.0, pctlrange0-1 \\
Shimmer and jitter & upleveltime75, upleveltime90
\end{tabular}

usually chosen. In this work, we used best-first search method.

\section{Emotion classification}

In this paper, we focused on four major emotion classes neutral, sad, happy, and angry, and did not include surprised, fear, and other classes in the classification experiments. We evaluated performances of support vector machine (SVM) with radial basis kernel function (SVM-RBF) implemented in the LIBSVM library [39] and Bayesian Networks (BayesNet) provided by the Weka pattern recognition tool $[40,41]$. The performance of SVM highly depends on the parameters used. In order to optimize the SVM performance, we used grid search with fivefold cross validation to select the penalty parameter for mislabeled examples $C$ and Gaussian parameter $\gamma$. We also linearly scaled each attribute to the range $[0,1]$. The scaling parameters for each attribute were calculated from the training data of each fold, and same scaling factors are applied to both corresponding training and testing data.

The performance of the classifiers was evaluated by tenfold cross-validation. To ensure the speaker independence, no instance of a test subject is allowed to be in the train dataset of each fold. For each experiment, the feature selection is performed using CFS to the training set of each fold. The results are presented in terms of confusion matrix, weighted average (WA) recall, and unweighted average (UA) recall. WA recall is defined as the ratio of the number of correctly classified instances to the total number of instances in the database. As classes are unbalanced in the databases, we also reported UA recall. UA recall is the average of per-class accuracies and more useful than WA recall when the distribution of classes is highly skewed.

\subsection{Categorical classification results}

First, we evaluated the relative importance of the seven low-level descriptors of acoustic features using BayesNet classifier. The performances are shown in Figure 9 in terms of UA recall. It can be seen that logarithmic power of Mel-frequency bands and mfcc seem to have more discriminative power than other LLDs.

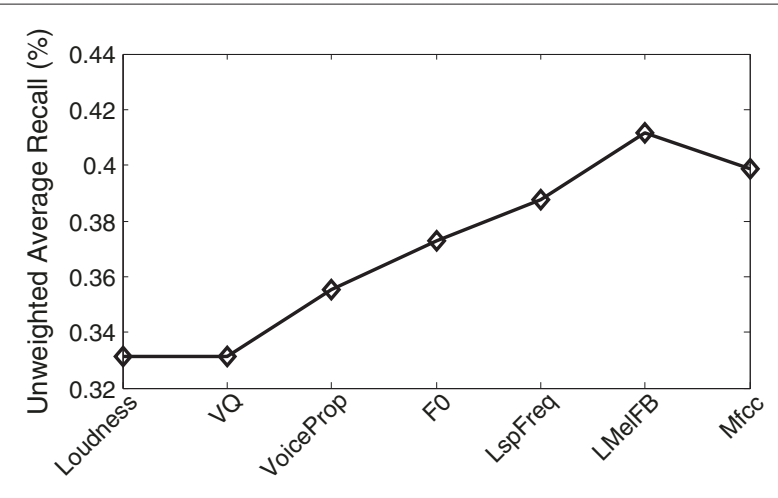

Figure 9 The classification performances of each LLD in terms of unweighted average recall. $V Q$, voice quality (jitter and shimmer); LMelFB, log mel frequency band.

The classification results are given in Table 5 in terms of confusion matrix. We obtain $70.9 \%, 26.8 \%, 13.1 \%$, and $62.4 \%$ recall rates using SVM and 48.5\%, 41.4\%, 22.5\%, and $69.7 \%$ recall rates using BayesNet for neutral, sad, happy, and angry classes, respectively. Overall, we obtain good recall rates for neutral, sad, and angry classes. However, the recognition accuracy for the happy class is about the chance level.

\subsection{Emotion primitives estimation}

Support vector regression $(\epsilon-\mathrm{SVR})$ with the radial basis kernel function [42] is used in this paper as it has shown promising results in performing emotion primitives estimation [19]. The $\epsilon-S V R$ design parameters, the complexity $C, \epsilon$, and the kernel parameter $\gamma$ are selected using grid-search with fivefold cross validation. For all regression experiments, LIBSVM [39] implementation of $\epsilon$-SVR was used. The performance of the estimators was evaluated in terms of correlation coefficient (CC), mean absolute error (MAE), and root-mean-square error (RMSE).

Table 5 Performances for categorical emotion classification

SVM-RBF BayesNet

Neutral Sad Happy Angry Neutral Sad Happy Angry

\begin{tabular}{|c|c|c|c|c|c|c|c|c|}
\hline Neutral & 1,524 & 205 & 133 & 288 & 1,042 & 485 & 271 & 352 \\
\hline Sad & 289 & 152 & 42 & 84 & 172 & 235 & 72 & 88 \\
\hline Happy & 218 & 39 & 53 & 94 & 122 & 66 & 91 & 125 \\
\hline Angry & 381 & 63 & 74 & 859 & 209 & 65 & 143 & 960 \\
\hline WA recall & \multicolumn{4}{|c|}{$57.5 \%$} & \multicolumn{4}{|c|}{$51.8 \%$} \\
\hline UA recall & \multicolumn{4}{|c|}{$43.3 \%$} & \multicolumn{4}{|c|}{$45.5 \%$} \\
\hline
\end{tabular}

The results are given in terms of confusion matrix, WA recall and UA recall for SVM (RBF kernel) and BayesNet classifiers. The diagonal elements (the values in italics) of confusion matrix represent the number of correctly predicted emotion labels. 

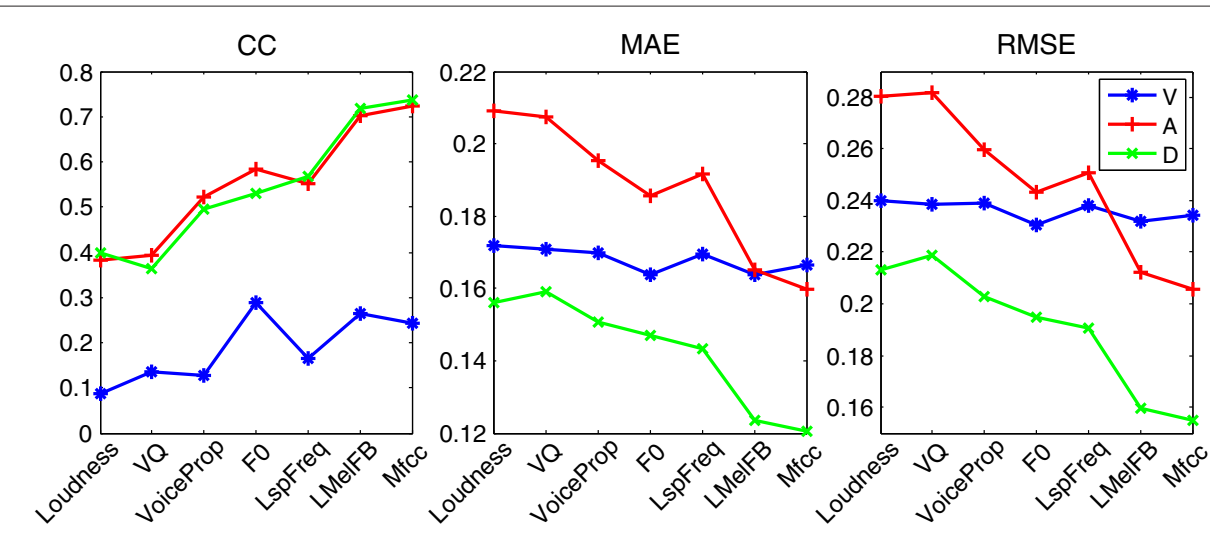

Figure 10 Performance comparisons of different acoustic feature groups for each emotion primitive for TURES database. Results are shown in terms of correlation coefficient (CC), mean absolute error (MAE), and root-mean-square error (RMSE).

$$
\begin{aligned}
& \mathrm{CC}=\frac{\sum_{i=1}^{N}\left(x_{i}-\bar{x}\right)\left(y_{i}-\bar{y}\right)}{\sqrt{\sum_{i=1}^{N}\left(x_{i}-\bar{x}\right)^{2}} \sqrt{\sum_{i=1}^{N}\left(y_{i}-\bar{y}\right)^{2}}} \\
& \text { MAE }=\frac{1}{N} \sum_{i=1}^{N}\left|x_{i}-y_{i}\right| \\
& \text { RMSE }=\sqrt{\frac{1}{N} \sum_{i=1}^{N}\left(x_{i}-y_{i}\right)^{2},}
\end{aligned}
$$

where $N$ is the number of speech data in the database, $x$ is the true value, and $y$ is the predicted value.

For the cross-corpus experiments, we employed the VAM database [11]. The VAM corpus consists of audiovisual recordings taken from German TV talk show called Vera-Am-Mittag. The corpus contains 946 spontaneous utterances from 47 participants of the show, and each utterance was labeled using a discrete five-point scale for three-dimensional emotion space of valence, activation, and dominance by 6 to 17 labelers.

\subsubsection{Estimation results}

First, we evaluated the relative importance of each LLD in emotion estimation. The performance comparisons of different acoustic feature groups for each emotion primitives using support vector regression are given for the TURES database in Figure 10. For this task, the $\epsilon$-SVR parameters $C, \gamma$, and $\epsilon$ were set to $1,0.01$, and 0.001 , respectively. The results are based on tenfold cross validation. As can be seen from Figure 10, for activation and dominance, mfcc are the most informative low-level descriptors followed by logarithmic power of Mel-frequency bands (LogMelFreqBand). For valence, F0, LogMelFreqBand and mfcc have more discriminative power than other LLD groups.

The regression results for emotion primitives are given in Table 6. The results show that all emotion primitives are estimated with a small mean absolute error, 0.166, 0.156 , and 0.119 for TURES database and $0.149,0.160$, and 0.153 for the VAM database, for valence, activation, and dominance, respectively. The regression results in terms of correlation coefficient show good results for activation and dominance for both databases. For valence, however, the correlation between the averaged ratings of the annotators (reference values) and the SVR estimates was low (only 0.288 for TURES and 0.310 for VAM).

Research shows that language and culture play an important role in how vocal emotions are perceived [43]. Recently, a few studies present results on cross-corpus evaluations, i.e., training on one and testing on a different one [34]. However, most of the work employed either different databases of the same language or Germanic

Table 6 The estimation performances for TURES and VAM databases

\begin{tabular}{lllllllrrrr}
\hline & & \multicolumn{3}{c}{ Valence } & \multicolumn{3}{c}{ Activation } & \multicolumn{3}{c}{ Dominance } \\
Train & Test & CC & MAE & RMSE & CC & MAE & RMSE & CC & MAE & RMSE \\
\hline TURES & TURES & 0.288 & 0.166 & 0.232 & 0.739 & 0.156 & 0.201 & 0.743 & 0.119 & 0.153 \\
VAM & TURES & 0.131 & 0.204 & 0.275 & 0.624 & 0.191 & 0.246 & 0.649 & 0.165 & 0.208 \\
VAM & VAM & 0.310 & 0.149 & 0.196 & 0.810 & 0.160 & 0.202 & 0.761 & 0.153 & 0.196 \\
TURES & VAM & 0.289 & 0.170 & 0.219 & 0.743 & 0.189 & 0.238 & 0.717 \\
\hline
\end{tabular}

Results are given in terms of correlation coefficient (CC), mean absolute error (MAE), and root-mean-square error (RMSE). The cross-corpus results for each primitive are also given. 


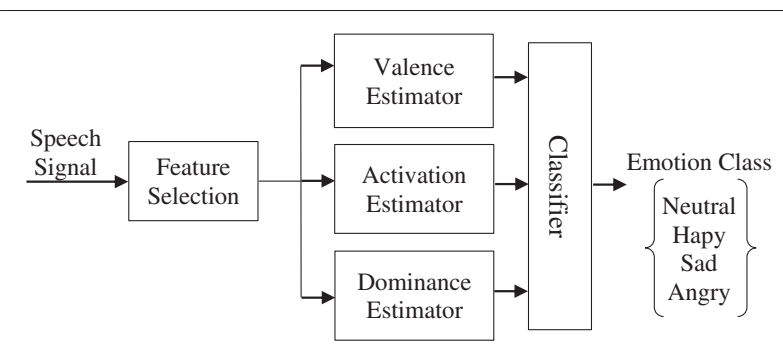

Figure 11 Block diagram of the approach to classify emotional classes using emotion primitives.

languages. Turkish is an agglutinative language, i.e. new words can be formed from existing words using a rich set of affixes. In this study, we performed cross-corpus experiments between the Turkish emotional speech database and the VAM corpus. The cross-corpus results were given in Table 6. It can be seen from the table that the crosscorpus training and testing seems to work especially for activation and dominance dimensions. For example, when TURES is chosen for training and VAM for testing, the correlation coefficients of 0.743 and 0.717 with 0.189 and 0.178 mean absolute errors were obtained for activation and dominance, respectively. For valence, like intra-corpus experiments, the cross-corpus results were not promising. This result indicates that acoustic information alone is not enough to discriminate emotions in valence dimension. These results are consistent with previous research [44]. Other sources of information, such as linguistic information, are needed in order to obtain better discrimination results in valence dimension [31].

\subsubsection{Emotion classification from the emotion primitives}

As a final experiment, we used the emotion primitives to classify emotion classes. A block diagram of the approach is shown in Figure 11. We used SVR to estimate the emotion primitives from acoustic features, and for the classification, BayesNet classifier is employed. First, we assumed perfect primitive estimation and used true labels as a feature set for training and testing the classifier. This experiment will show the upper bound of the classification performance. Table 7 shows that a recognition rate of $77.6 \%$ in terms of UA recall can be achieved using true emotion primitive values. However, when we used regression estimates, the classification performance is about random. The underlying reason is the low regression performance for the valence dimension.

\section{Conclusion}

In this work, we carried out a study on emotion recognition from Turkish speech using acoustic features. In recent years, several corpora in different languages have been collected; however, Turkish is not among the languages that has been investigated in the context of emotion recognition. In this paper, we presented the Turkish Emotional Speech Database and reported the baseline results. Categorical representations and dimensional descriptions are two common approaches to define emotion present in speech. In categorical approach, a fixed set of words is used to describe an emotional state, whereas in the dimensional approach, emotion is defined as points in the multidimensional space. The three most common dimensions used are valence, activation, and dominance which represent the main properties of emotional states. In this work, both categorical evaluation and emotion primitive estimation were performed. An unweighted average recall of $45.5 \%$ was obtained for the classification. For emotion dimension estimation, the regression results in terms of correlation coefficient are promising for activation and dominance, with 0.739 and 0.743, respectively. For valence, however, the correlation between the averaged ratings of the evaluators (reference values) and the SVR estimates was low (only 0.288). In this study, we also performed cross-corpus evaluations, and the results were promising especially for activation and dominance dimensions. This result indicates that acoustic information alone is not enough to discriminate emotions in valence dimension. Future work includes the use of linguistic information to improve the classification and regression results especially for valence.

Table 7 Emotion classification results from three-dimensional emotion primitives

\begin{tabular}{lcccccccc}
\hline & Neutral & Sad & Happy & Angry & Neutral & Sad & Happy & Angry \\
\hline Neutral & 1,955 & 53 & 57 & 85 & 1,430 & 24 & 0 & 696 \\
Sad & 114 & 342 & 1 & 110 & 371 & 11 & 0 \\
Happy & 93 & 0 & 302 & 9 & 262 & 6 & 1 \\
Angry & 163 & 50 & & 2 & 1,162 & 947 & 13 & 0 \\
WA recall & & & $83.6 \%$ & & & $41.3 \%$ & \\
UA recall & & $77.6 \%$ & & & & $24.8 \%$ & \\
\hline
\end{tabular}

Left confusion matrix: emotion classification from the ground truth emotion primitives. Right confusion matrix: emotion classification from the estimates of emotion primitives. SVR is used to estimate the three emotion primitives. For classification, BayesNet classifier is used. The values in italics of the confusion matrices represent the number of correctly predicted emotion labels. 


\section{Endnotes}

a The Turkish emotional speech database is available to the research community through the website http://www. turesdatabase.com.

\section{Competing interests}

The authors declare that they have no competing interests.

\section{Acknowledgements}

This work was supported by the Turkish Scientific and Technical Research Council (TUBITAK) under project no. 109E243.

\section{Received: 14 May 2013 Accepted: 14 October 2013}

Published: 5 December 2013

\section{References}

1. F Burkhardt, A Paeschke, M Rolfes, W Sendlmeier, B Weiss, A database of, German emotional speech. Paper presented at the Interspeech 9th European conference on speech, communication and technology. Lisbon, Portugal, 4-8 Sept 2005

2. IS Engberg, AV Hansen, Documentation of the Danish Emotional Speech Database. (Aalborg University, Aalborg, 1996)

3. M Liberman, K Davis, M Grossman, N Martey, J Bell, Emotional Prosody, Speech and Transcripts. (Linguistic Data Consortium, Philadelphia, 2002)

4. T Banziger, M Mortillaro, K Scherer, Introducing the Geneva multimodal expression corpus for experimental research on emotion perception. Emotion. 12, 1161-1179 (2012)

5. E Douglas-Cowie, R Cowie, I Sneddon, C Cox, O Lowry, M Mcrorie, J Claude Martin, L Devillers, S Abrilian, A Batliner, N Amir, K Karpouzis, in Affective Computing and Intelligent Interaction: Lecture Notes in Computer Science, ed. by ACR Paiva, R Prada, and RW Picard. The HUMAINE Database: addressing the collection and annotation of naturalistic and induced emotional data, vol. 4738 (Springer Berlin, 2007), pp. 488-500

6. B Schuller, B Vlasenko, F Eyben, G Rigoll, A Wendemuth, in IEEE Workshop on Automatic Speech Recognition Understanding. Acoustic emotion recognition: a benchmark comparison of performances (IEEE Merano, Italy, 13 Nov-17 Dec 2009)

7. JHL Hansen, S Bou-Ghazale, Getting started with SUSAS: a speech under simulated and actual stress database. Paper presented at the fifth European conference on speech communication and technology, EUROSPEECH 1997. Rhodes, Greece 22-25 Sept 1997

8. C Busso, M Bulut, CC Lee, A Kazemzadeh, E Mower, S Kim, J Chang, S Lee, S Narayanan, IEMOCAP: Interactive emotional dyadic motion capture database. J. Lang. Resour. Eval. 42(4), 335-359 (2008)

9. G McKeown, M Valstar, R Cowie, M Pantic, in IEEE ICME. The SEMAINE corpus of emotionally coloured character interactions (Suntec City, 19-23 Jul 2010)

10. S Steidl, Automatic Classification of Emotion Related User States in Spontaneous Children's Speech. (University of Erlangen-Nuremberg, Germany, 2009)

11. M Grimm, K Kroschel, S Narayanan, in IEEE International conference on multimedia and expo (ICME). The Vera am Mittag German audio-visual emotional speech database (IEEE Hannover, Germany, 23 Jun-26 Apr 2008)

12. C Clavel, I Vasilescu, L Devillers, T Ehrette, G Richard, in LREC. The SAFE Corpus: fear-type emotions detection for surveillance applications (Genoa, Italy, 24-26 May 2006)

13. E Douglas-Cowie, N Campbell, R Cowie, P Roach, Emotional speech: towards a new generation of databases. Speech Commun. Spec. Issue, Speech and Emotion. 40, 33-60 (2003)

14. P Ekman, in Handbook of Cognition and Emotions, ed. by L Dalgleish, M Power. Basic emotions (Wiley New York, 1999), pp. 409-589

15. E Douglas-Cowie, R Cowie, M Schroder, in ISCA Workshop on speech and emotion. A new emotion database: considerations, sources and scope (Newcastle UK, 5-7 Sept 2000)

16. J Ang, R Dhillon, A Krupski, E Shriberg, A Stolcke, in ICSLP 2002. Prosody-based automatic detection of annoyance and frustration in human-computer dialog (ISCA Denver, Colorado, 16-20 Sept 2002)

17. TL Nwe, SW Foo, L De Silva, Speech emotion recognition using hidden Markov models. Speech Commun. 41(4), 603-623 (2003)
18. CM Lee, S Narayanan, Towards detecting emotions in spoken dialogs. IEEE T Speech Audi. P. 13(2), 293-303 (2005)

19. M Grimm, K Kroschel, E Mower, S Narayanan, Primitives-based evaluation and estimation of emotions in speech. Speech Commun. 49, 787-800 (2007)

20. BS Schuller, A Batliner, D Seppi, S Steidl, T Vogt, J Wagner, L Devillers, L Vidrascu, N Amir, L Kessous, V Aharonson, in eighth conference on InterSpeech. The relevance of feature type for the automatic classification of emotional user states: low level descriptors and functionals (ISCA Antwerp, Belgium, 27-31 Aug 2007)

21. C Clavel, I Vasilescu, L Devillers, G Richard, T Ehrette, Fear-type emotion recognition for future audio-based surveillance systems. Speech Commun. 50(6), 487-503 (2008)

22. S Yildirim, S Narayanan, A Potamianos, Detecting emotional state of a child in a conversational computer game. Comput. Speech and, Lang. 25, 29-44 (2011)

23. EM Albornoz, DH Milone, HL Rufiner, Spoken emotion recognition using hierarchical classifiers. Comput. Speech and Lang. 25(3), 556-570 (2011)

24. CC Lee, E Mower, C Busso, S Lee, S Narayanan, Emotion recognition using a hierarchical binary decision tree approach. Speech Commun. 53(9-10), 1162-1171 (2011). [Special issue: Sensing Emotion and Affect - Facing Realism in Speech Processing]

25. T Polzehl, A Schmitt, F Metze, M Wagner, Anger recognition in speech using acoustic and linguistic cues. Speech Commun. 53(9-10), 1198-1209 (2011)

26. A Batliner, S Steidl, B Schuller, D Seppi, T Vogt, J Wagner, L Devillers, L Vidrascu, V Aharonson, L Kessous, N Amir, Whodunnit - searching for the most important feature types signalling emotion-related user states in speech. Comput. Speech Lang. 25, 4-28 (2011)

27. F Eyben, M Wöllmer, B Schuller, in international conference on multimedia. openSMILE: the Munich versatile and fast open-source audio feature extractor (ACM Firenze, Italy, 25-29 Oct 2010)

28. S Arunachalam, D Gould, E Andersen, D Byrd, S Narayanan, in InterSpeech. Politeness and frustration language in child-machine interactions (Aalborg Denmark, 3-7 Sept 2001)

29. A Batliner, S Steidl, B Schuller, D Seppi, K Laskowski, T Vogt, L Devillers, L Vidrascu, N Amir, L Kessous, V Aharonson, in fifth Slovenian and first international language technologies conference. Combining efforts for improving automatic classification of emotional user states (IS-LTC'06 Ljubljana, Slovenia, 9-10 Oct 2006)

30. B Schuller, A Batliner, S Steidl, D Seppi, in IEEE international conference on acoustics, speech, and signal processing. Emotion recognition from speech: putting ASR in the loop (IEEE Taipei, Taiwan, 19-24 Apr 2009)

31. B Schuller, Recognizing affect from linguistic information in $3 D$ continuous space. IEEE Trans. Affect. Comput. 2(4), 192-205 (2012)

32. B Schuller, S Steidl, A Batliner, F Burkhardt, L Devillers, C Muller, $S$ Narayanan, in InterSpeech. The INTERSPEECH 2010 paralinguistic challenge (Makuhari Japan, 26-30 Sept 2010)

33. M Shami, W Verhelst, in Speaker Classification II LNCS, ed. by C Müller. Automatic classification of expressiveness in speech: a multi-corpus study (Springer Berlin, 2007), pp. 43-56

34. B Schuller, B Vlasenko, F Eyben, M Wollmer, A Stuhlsatz, A Wendemuth, G Rigoll, Cross-corpus acoustic emotion recognition: variances and strategies. IEEE Trans. Affect. Comput. 1(2), 119-131 (2010)

35. C Oflazoglu, S Yildirim, in IEEE 19th conference signal processing and communications applications. Turkish emotional speech database (IEEE Antalya, Turkey, 20-22 Apr 2011)

36. J Fleiss, Measuring nominal scale agreement among many raters. Psychol. Bull. 76(5), 378-382 (1971)

37. M Bradley, PJ Lang, Measuring emotion: the self-assessment manikin and the semantic differential. J. Behav. Ther. Exp. Psychiatry. 25, 49-59 (1994)

38. M Hall, Correlation-based feature selection for machine learning. (PhD thesis, University of Waikato, New Zealand, 1999)

39. CC Chang, CJ Lin, LIBSVM: a library for support vector machines. ACM Trans. Intell. Syst. Technol. 2, 1-27 (2011)

40. M Hall, E Frank, G Holmes, B Pfahringer, P Reutemann, IH Witten, The WEKA data mining software: an update. SIGKDD Explor. Newsl. 11, 10-18 (2009)

41. R Bouckaert. Bayesian Network Classifiers in Weka for Version 3-5-7, Technical Report (Waikato University Hamilton, NZ, 2008) 
42. AJ Smola, B Schölkopf, A tutorial on support vector regression. Stat. Comput. 14(3), 199-222 (2004)

43. KR Scherer, R Banse, $\mathrm{H}$ Wallbott, Emotion inferences from vocal expression correlate across languages and cultures. J Cross Cult, Psychol. 32, 76-92 (2001)

44. M Grimm, K Kroschel, S Narayanan, in IEEE international conference on acoustics, speech and signal processing. Support vector regression for automatic recognition of spontaneous emotions in speech (Honolulu, HI, 15-20 Apr 2007)

doi:10.1186/1687-4722-2013-26

Cite this article as: Oflazoglu and Yildirim: Recognizing emotion from Turkish speech using acoustic features. EURASIP Journal on Audio, Speech, and Music Processing 2013 2013:26.

\section{Submit your manuscript to a SpringerOpen ${ }^{\circ}$ journal and benefit from:}

- Convenient online submission

Rigorous peer review

- Immediate publication on acceptance

- Open access: articles freely available online

- High visibility within the field

- Retaining the copyright to your article

Submit your next manuscript at $\boldsymbol{\triangleright}$ springeropen.com 